\title{
Authenticating Features in the TEI
}

Sean M. Winslow

\section{OpenEdition}

\section{Journals}

Electronic version

URL: https://journals.openedition.org/jtei/3608

DOI: 10.4000/jtei.3608

ISSN: 2162-5603

\section{Publisher}

TEl Consortium

Electronic reference

Sean M. Winslow, "Authenticating Features in the TEI", Journal of the Text Encoding Initiative [Online], Issue 13 | May 2020 - November 2022, Online since 23 September 2021, connection on 24 November 2022. URL: http://journals.openedition.org/jtei/3608 ; DOI: https://doi.org/10.4000/jtei.3608

For this publication a Creative Commons Attribution 4.0 International license has been granted by the author(s) who retain full copyright. 


\title{
Authenticating Features in the TEI
}

\author{
Sean M. Winslow
}

\section{ABSTRACT}

Textual and paratextual elements that serve to establish the authenticity of documents abound and carry meaning that is important-often central-to the meaning of the document as received, but are currently only lightly modeled in TEI. This paper will discuss issues in the modeling of authenticating features across time periods and regional boundaries, from the existing <seal> element to medieval European chirographs to Japanese jitsu-in. Here, I will make the case for the applicability of authenticating elements, introduce a preliminary working vocabulary of methods of authenticating elements, and demonstrate how they can be modeled in TEI.

\section{INDEX}

Keywords: authentication, seal, stamp, authenticity, diplomatics, charters, 


\section{Introduction}

1 Textual and paratextual elements that serve to establish the authenticity of documents abound and carry authority that is important-often central-to the meaning of the document as received. An authentic document from a legitimate sender issuing an order, for example, is completely different in interpretation from an inauthentic copy of an order, although the reception may change based upon whether it was perceived by the recipients as authentic. Issues related to authentication are currently only lightly modeled in TEI. This paper will discuss issues in the modeling of authenticating features across time periods and regional boundaries, from the existing $<$ seal> element to medieval European chirographs to Japanese jitsu-in. Here, I will make the case for the applicability of authenticating elements, introduce a preliminary working vocabulary of methods of authenticating elements, and demonstrate how they can be modeled in TEI.

The present use case arises out of the ongoing work of mapping of medieval documents marked up by the http://www.cei.Imu.de/Charters Encoding Initiative (CEI), ${ }^{1}$ a charters-data-specific fork of TEI P4 with its own elements and structures and separate development history, to TEI P5. Georg Vogeler, who began the CEI, wondered what a model that encompassed both seals and digital hashes might look like and initiated the current discussion of authentication and the current ODD ("One Document Does it all”) extension. This discussion and the ODD extension are part of the Austrian Science Fund (FWF) Open Research Data (ORD) Project 84, Retain Domain Specific Functionalities in a Generic Repository with Humanities Data, for which I worked as a postdoctoral research associate. Vogeler's observation, and the model of the CEI, were the genesis of the current discussion of authentication and the current extension, and the present author would like to acknowledge Georg Vogeler's advice and feedback throughout the process. ${ }^{2}$ In the process of modeling medieval documents for the update of CEI to TEI P5, it was necessary to address such issues as they occur in the context of medieval documents, with elements like chirographs, monograms, and lists of witnesses. The practices modeled in the CEI, however, are similar across time periods and regions, with little clear demarcation in the exact differences in form and function of seals, stamps, monograms, jitsu-in, and rotas: all of these practices exist within a larger spectrum of practice that spans time and culture, from ancient Mesopotamia to medieval England to modern Japan. Accordingly, this paper considers not just medieval charters and documents currently being encoded for the CEI, but also potential needs for the modeling of 
related materials and of items like text-bearing museum objects that have authentication features, such as banknotes and passports. Born-digital objects such as email likely need further, specialized attention in the future, and are beyond the scope of the present study. The paper introduces a dedicated element for authentication, based upon (and expanding) current practice in the CEI. This paper attempts to generalize the markup of authenticating elements encountered through diplomatic study of and markup of medieval documents, moving beyond the original disciplinary context of the originating project. The approach here is very different from that taken by SigiDoc (Sopracasa 2020), a recent offshoot of EpiDoc, which takes a rather numismatic approach, focusing on seals as individual objects, independent of any accompanying materials, a decision derived from the conditions of Byzantine sigillography, where scarcely any documents survive. It also differs from other projects that contain elements describing authentication (e.g., Nomisma ${ }^{3}$ ) which are discipline-specific (i.e., numismatic) in approach, in aiming for generalizable universality.

\section{Prior Art: Manuscript Catalogues and Diplomatics}

4 Diplomatics, the science of the interpretation of charters and other documentary materials, has since its inception been concerned with the authenticity of documents. Foundational episodes in the history of the discipline center on this concern: Lorenzo Valla's debunking of the Donation of Constantine set the model that diplomatists have generally followed, that of the expert making decisions based upon the totality of the evidence about the content and context of a historical document to determine whether it is authentic or a forgery (Valla 1440). When Jean Mabillon laid the groundwork for the modern discipline in his De re diplomatica libri VI, he did so in response to competing claims about the authenticity of documents held in the abbey of Saint-Denis (Mabillon 1681). His six-year-long program to establish the authentic nature of those documents led to the foundation of the disciplines of paleography and diplomatics and also set the model for the learned diplomatist who approaches historical documents in a holistic way to deliver expert and authoritative judgments on the authenticity of historical documents. Diplomatics as a discipline is one of the auxiliary sciences of history (Historische Hilfswissenschaften), and exists as both a general tool for historians and a specialist field of inquiry on its own. In its modern form, it sees significant use in archival cataloging of documents. While the TEI manuscript description (msdescription) module provides many elements for representing traditional catalog 
elements in TEI (Cummings 2017), these cataloging elements are highly focused on books, not documents. Not only do documents have their own forms, but they also have the specific trait of being anchored in a legal context: a document which is treated entirely as text without reference to its authentic status can be misleading to the modern historian, as treating an inauthentic document as if it were authentic (i.e, with the force of law behind it) leads the modern scholar down a false historical path. ${ }^{4}$ Traditionally, authentication has been a matter for an expert, who makes a decision based upon the totality of the form and content of the document, processes it through personal expertise in the relevant historical and legal contexts, and makes a pronouncement on whether it is authentic or inauthentic. ${ }^{5}$

Ideally, TEI would make room for the inclusion of specialist expert knowledge and judgment, but at the same time, as content-based markup, de-link that judgment from the real, present elements which are evidence for authentication. This separates the text, as an object that can be freely transcribed, edited, and forwarded, from the images, which can be reproduced through photographs; from the authenticating value of those textual or image elements; and also from the document itself, the authenticity of which can only be determined by the kind of connoisseurship which comes from long experience and exposure to documents. In this way, a combination of authentic and inauthentic, authenticating and nonauthenticating elements can be separated from the overall document (which, in the case that it contains a mix of authentic and inauthentic authentication elements, is likely itself inauthentic). That authenticating elements bear significance beyond their text or image and convey information that is on par with the text itself is well established (Rück 1996). This paper suggests a way to regularly mark up such elements using an extension of TEI, with a suggested preliminary controlled vocabulary useful as a target for subtype pointers.

\section{Authenticating Features}

7 Seals, whether affixed (applied directly to the document) or pendant (hanging from the document from a cord or parchment/paper tag), served to express that the document had been legitimately and authentically issued by the owner of the seal. ${ }^{6}$ Seal matrices were physical devices with complex carvings on them, presenting both a material and an artistic/technological barrier to forgery, and therefore serving as an attestation of the authentic nature of the document to which 
they were attached. But seals are not specific to the format enshrined in the TEI Guidelines, the medieval wax seal: its predecessors, circular seals meant for rolling in clay, are much attested from the ancient Middle East, and using signet rings for personal attestation was a common practice from the Ancient Middle East ${ }^{7}$ through the Middle Ages, with variances in fashion, until going out of general use in the nineteenth century.

8 Though wax seals have a physical and artistic character that can be described with the relevant TEI elements, their intended purpose points to their value as paratextual elements, as their authenticating value is independent of their value as art or as text. Accordingly, it makes sense that the authenticating value needs highlighting, and the $<$ sealDesc $>$ and $<$ seal $>$ elements largely handle these concerns, bundling the various aspects of seals-their artistic description, physical character, prosopographical attributes, and other paratextual qualities-in one place, representing the plural modes of communication that seals are involved in. But do seals in themselves represent the full range of features that similarly combine textual, artistic, and paratextual elements for the purpose of authenticating documents?

9 TEI P5 provides the elements <seal $>$ and $<$ sealDesc $>$ as part of the msdescription module. A closely related feature of manuscripts which is also modeled is <stamp $>$. Stamps, while differing in material form, often have similar (if not identical) visual arrangement, incorporating elements of text and heraldry in round or pointed oval seals, directly borrowing their visual forms from such seals; in fact, there is little to distinguish a single-matrix affixed seal (one matrix, pressed into hot wax applied to the page) from the impression of an ink stamp (one stamp, pressed into ink applied to the page). Historically, they have been largely interchangeable, with stamps taking over as authenticating features and still widely used by government and corporate offices, as well as for indicating ownership (as in the Bodleian stamp, figure 1). Stamps outside of Europe have a long history of use in ways that are directly analogous to the use of seals in Europe, with widespread use in the Middle East and East Asia; when discussing the latter, the term seal refers to inked stamps (jitsu-in in Japan and colloquially "chops" in China) used to sign and authenticate documents. Despite the similar ways that (wax) seals and (inked) seals/stamps operate, at present TEI treats them quite differently. Inked seals/stamps are treated as text-level elements, placed where they appear in the running text, unlike <seal>s, which are considered to be part of the physical description of the document, within msDesc/physDesc/sealDesc/seal, although seals often 
contain text themselves (since <msDesc $>$ is catalog-level information, text in seals is currently treated as a description of the seal, not as text, making text in seals problematic to properly capture). Examples 1 and 2 show how they are currently described.

Example 1. Stamps: The example in the TEI P5 documentation for stamp ("Watermarks and Stamps," TEI Consortium 2019, sec. 10.3.3, https://tei-c.org/Vault/P5/3.6.0/doc/tei-p5-doc/en/html/MS.html\#mswat $<$ rubric $>$

$<$ lb/>Apologyticu TTVLLIANI AC IGNORATIA IN XPO IHV <lb/>SI NON LICET <lb/>NOBIS

RO <lb/>manii imperii <stamp>Bodleian stamp</stamp $>$

$<\mathrm{lb} />$

$</$ rubric $>$

Example 2. Seals. The example in the TEI P5 documentation for seal ("Seals," TEl Consortium 2019, sec. 10.7.3.2, https://tei-c.org/Vault/P5/3.6.0/doc/tei-p5-doc/en/html/MS.html\#msphse).

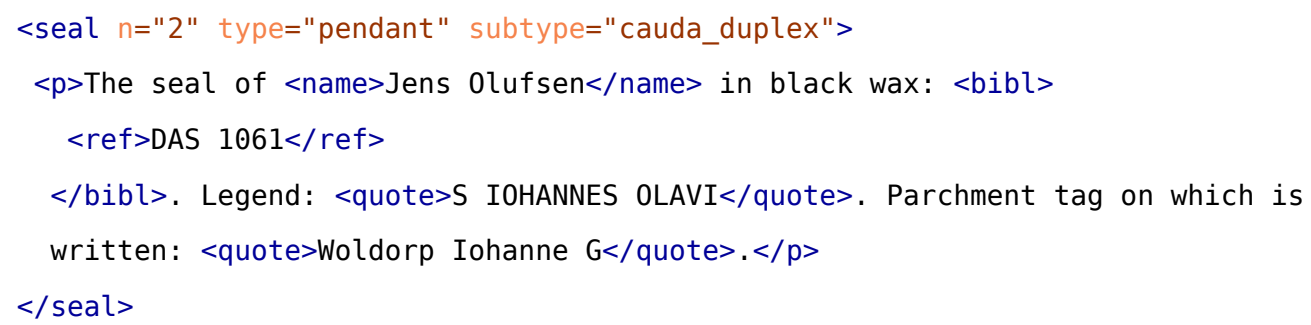

Figure 1. An example Bodleian stamp.

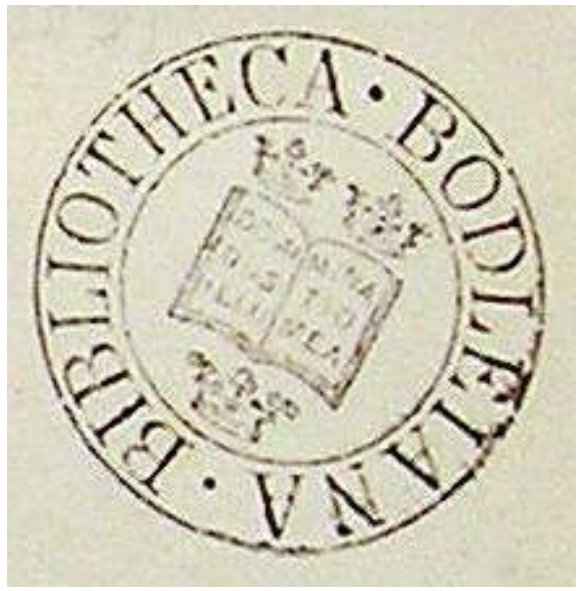


Image from Wikimedia.https://commons.wikimedia.org/wiki/File:Bibliotheksstempel_Bodleiana.jpg

Note that despite being part of the running text of the document, the content of the stamp element in example 1 is not text. Presumably, in this case, it should read "BIBLIOTHECA BODLEIANA" as seen in figure 11 (and, if the writing in the Coat of Arms of the University of Oxford is included, also "DOMINUS ILLUMINATIO MEA"), but instead the example is a text reference to the identity of the stamp (although, since there have presumably been multiple Bodleian stamps over the years, a rather insufficient one). In this sense, the content of the <stamp> element is rather parallel to the content of the <seal > element, despite the actual content models being quite different (<seal>, which may contain model.pLike and <decoNote>, only serves as a direct container for three different elements; <stamp>, which may contain macro. phraseseq, serves as a container for over 100, but none of the same three that <seal $>$ contains: $<$ decoNote $>,<p>$, and $<a b>$ ). ${ }^{8}$

\section{Authenticating Features Not Covered by the TEl: Medieval Examples}

As explained above, despite the differences in modeling in TEI P5, seals and stamps often-though not always-perform the same or similar functions, while having the same or similar layouts and very similar physical forms. But these are far from the only authenticating forms, as medieval documents also used split media (chirograph, poll, indenture, or tally), witnessing, various forms of signing (crosses, rotas, and monograms), reproduction/publication (vidimus, etc.), and less easily catalogued features, like specific chancellery styles of documents and handwriting (most notably, the ornate papal chancellery style). All of these elements served to establish that a document was what it claimed to be, and that the contents of the document had the force of law behind them. A legal document marked up without attention to these features is just a text (generally a very formulaic one, written from a model), and missing a crucial and inseparable aspect of its value to its producers and recipients: its status as a legal object. Compare, for example, the XML representation of document 570411a from the Monumenta Germaniae Historica (http://telota.bbaw.de/ constitutiones/data/texts/570411a.xml) ${ }^{9}$ with its image (http://telota.bbaw.de/constitutiones/ resources/images/Urkunde.gif. Though the document does mention a "Monogramm," it is not

Journal of the Text Encoding Initiative, Issue 13, 2021-09-23 Selected Papers from the 2018 TEI Conference 
marked up with any specialized element, and both the monogram and the half of the seal that remains-both bearing text and crucial for establishing that the accompanying text is authenticare not treated as text or with the care with which text would be treated.

Figure 2. Rota of Pope Innocent II in a charter, second graphic of Chartularium Sangallense 03 (1004-1277) Nr. 898, S. 21-22, accessed July 2, 2021, https://www.monasterium.net/mom/CSGIII/Nr_898_S_21-22/charter

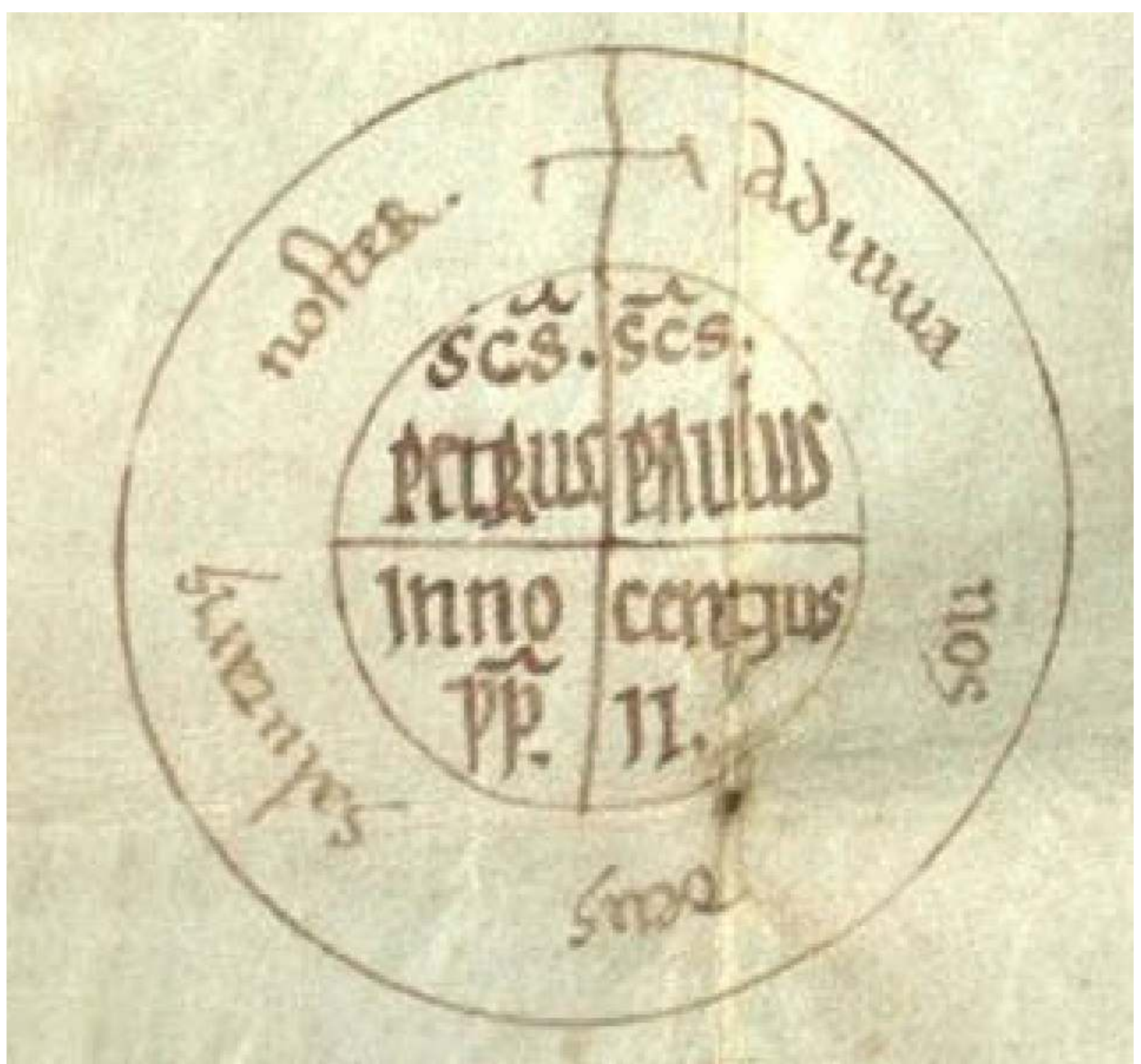


Figure 3. Leaden bull attached to a charter, second graphic of Chartularium Sangallense 03 (1004-1277) Nr. 898, S. 21-22, accessed July 2, 2021, https://www.monasterium.net/mom/CSGIII/Nr_898_S_21-22/charter.

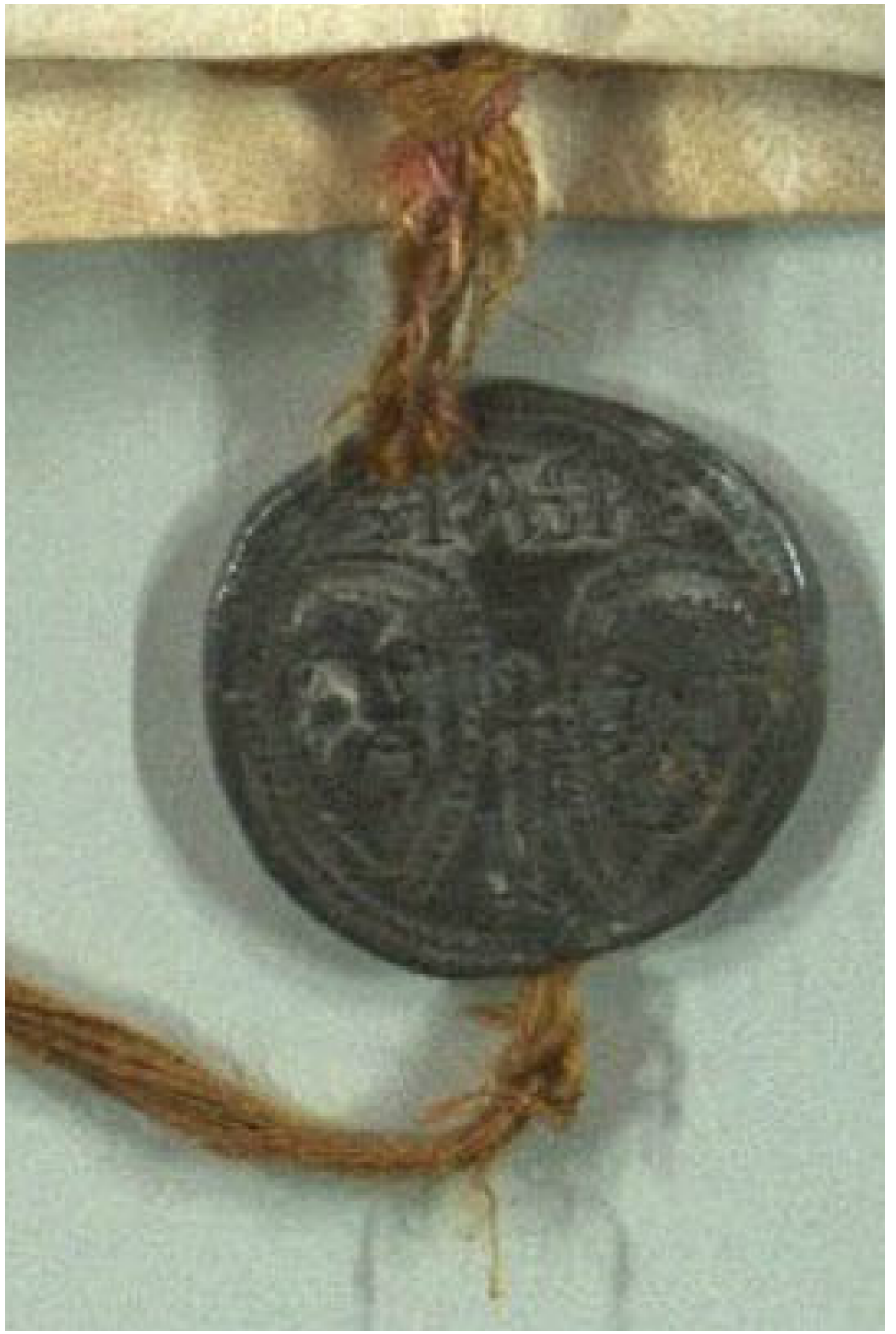

Journal of the Text Encoding Initiative, Issue 13, 2021-09-23 Selected Papers from the 2018 TEI Conference 
Figure 4. Bene Valete in a charter, second graphic of Chartularium Sangallense 03 (1004-1277) Nr. 898, S. 21-22, accessed July 2, 2021, https://www.monasterium.net/mom/CSGIII/Nr_898_S_21-22/charter

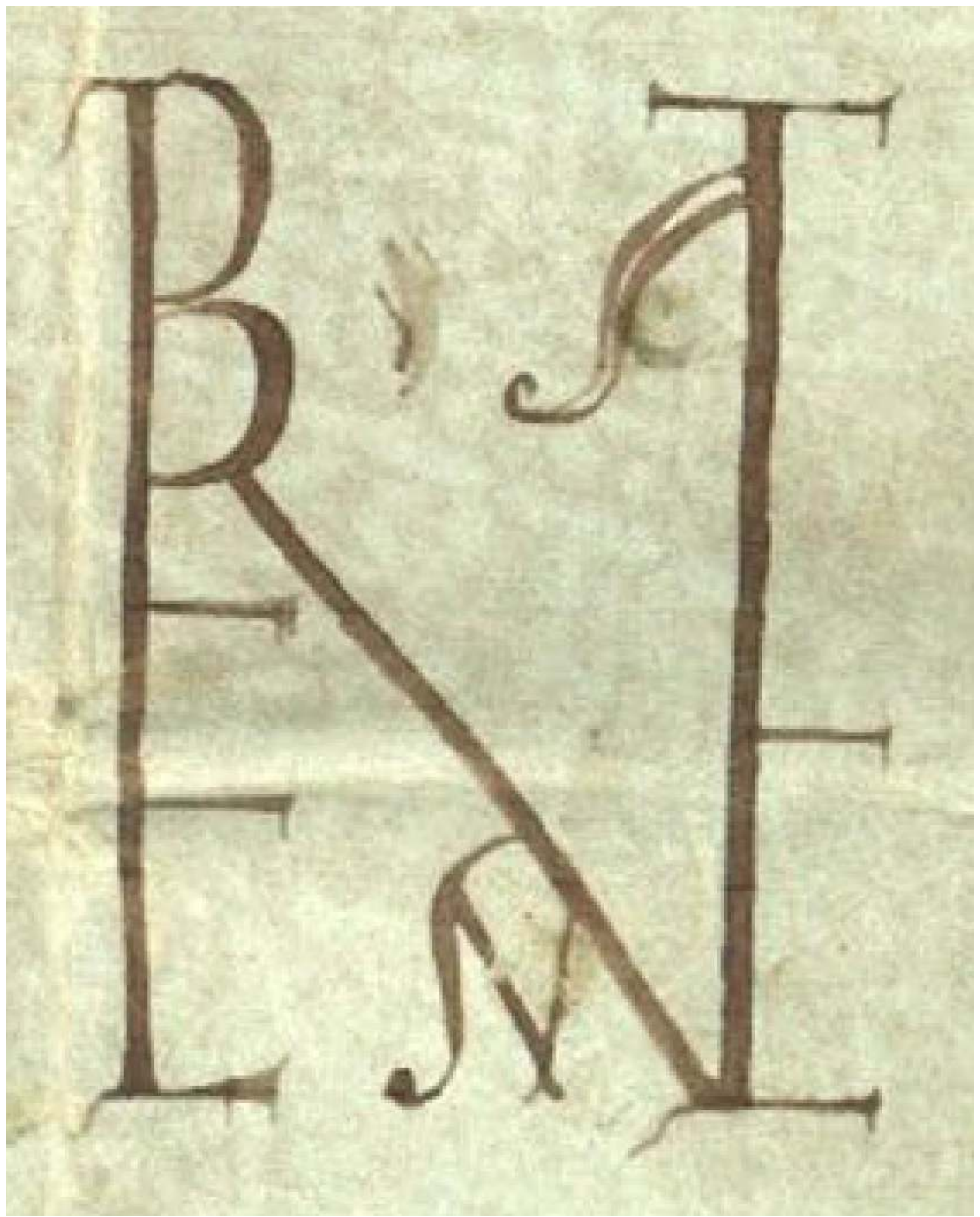

Journal of the Text Encoding Initiative, Issue 13, 2021-09-23 Selected Papers from the 2018 TEI Conference 
Figure 5. Subscription of Pope Innocent II in a charter, second graphic of Chartularium Sangallense 03 (1004-1277) Nr. 898, S. 21-22, accessed July 2, 2021, https://www.monasterium.net/mom/CSGIII/ Nr_898_S_21-22/charter

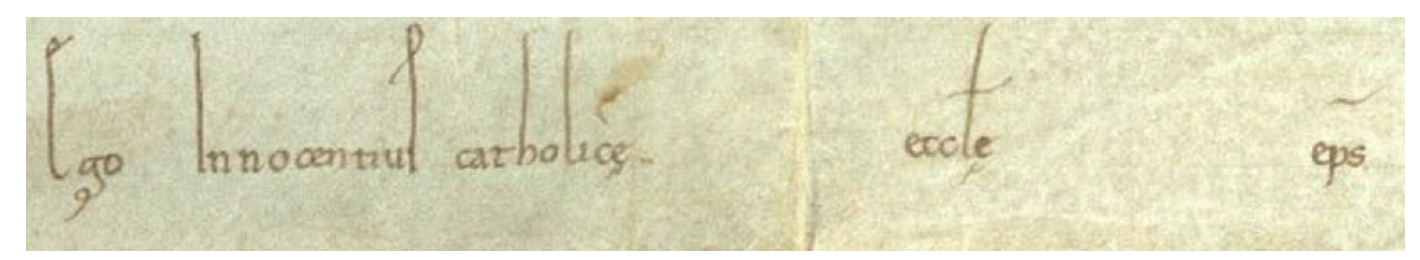

13 A feature that is currently not specifically modeled at all in TEI, but which serves a similar purpose to both stamp and seal, and even appears similar to both, is a rota or monogram. Both are handwritten or hand-drawn devices involving the names or initials of the issuer of the document, formalized enough to be recognizable as separate from other forms of signing. A rota is a specific, papal form of the monogram, which is characteristic enough to be described using its own term and structured similarly to a stamp (see figure 2). Similarly to the stamp and seal above, the rota contains both textual and figural elements, arranged in a formalized and consistent way, serving to establish the authenticity of the sender of the document. The round format of the rota and the consistent styling of the materials within directly invoke the style of seals or stamps, and contain notable similarities with the specific papal version of the seal, the leaden bull (figure 3).

Extending the idea of a drawn device that represents the person and authority of the drafter, the notarial signet is a related conventional symbol in the form of a drawing (often with writing) that stands in for the person preparing or acting in a notarial authority for the document. A similar element, unique to the papal chancellery, is the Bene Valete (figure 4), which is structured like a monogram and accompanies the other elements of papal authentication, like the rota and signature as well as the bull (papal seal, figure 3).

15 All of these features are supplemented (or, in many modern European and American cases, replaced) by the autograph signature (as pictured in figure 5), but that is far from the only version of signing used historically, and the cross, and in some cases fingerprints, used for signing work equivalently to indicate consent to the terms of a document. Indeed, seals, stamps, signatures, and crosses all have overlapping uses for asserting assent. Crosses were commonly used by witnesses to documents when the witnesses did not attach their own seals. Crosses overlap with signatures for the illiterate, and did so into the recent past. Stamps used for signing are still current in East 
Asia and coexist with signatures on documents throughout the world, often asserting that the person signing has the authority to sign on behalf of an organization or institution, represented by the stamp. An interesting case of the coexistence of stamps and crosses and signatures was documented by Ramble (2018), who additionally finds inked fingerprints thrown into the mix. He tracks changes in the documentary practice of the Mustang region of Nepal from inked stamps (associated with Tibetan practices) to fingerprints (associated with Indian, perhaps British Raj, practice), these practices corresponding to a movement from Tibetan to Nepali legal terms. Fingerprints (and handprints, in some examples) can be clearly seen to replace stamps on a onefor-one basis, demonstrating their analogous authentication value. ${ }^{10}$

16 As the above examples show, there are a range of technologies and practices which bleed together both in their place on a visual spectrum and in their practical use, and can thus be productively classified together, serving a similar function to seals, often with similar forms, but not yet described by the TEI. Other less obviously similar practices also serve a similar function, which we can easily infer through their co-use in service to proving the authenticity of documents, though they may not immediately be visually similar. An interesting medieval example is what I will call "split medium" authentication, and includes a number of related practices: chirographs, polls, indentures, and tally sticks. 


\section{Beyond Seals and Stamps: Medium as Authentication}

Figure 6. Chirograph from 1177, Departmental Archives of Val-d'Oise, part of the virtual exhibition Ciro graphum, un charte exceptionnelle de 1177 (http://archives.valdoise.fr/expositions/exposition-ciro-graphumune-charte-exceptionnelle-de-3/n:213). Paris, Archives nationales, AE/II/181 digitally reunited with Pontoise, Archives départementales du Val-d'Oise, 9 H 81.

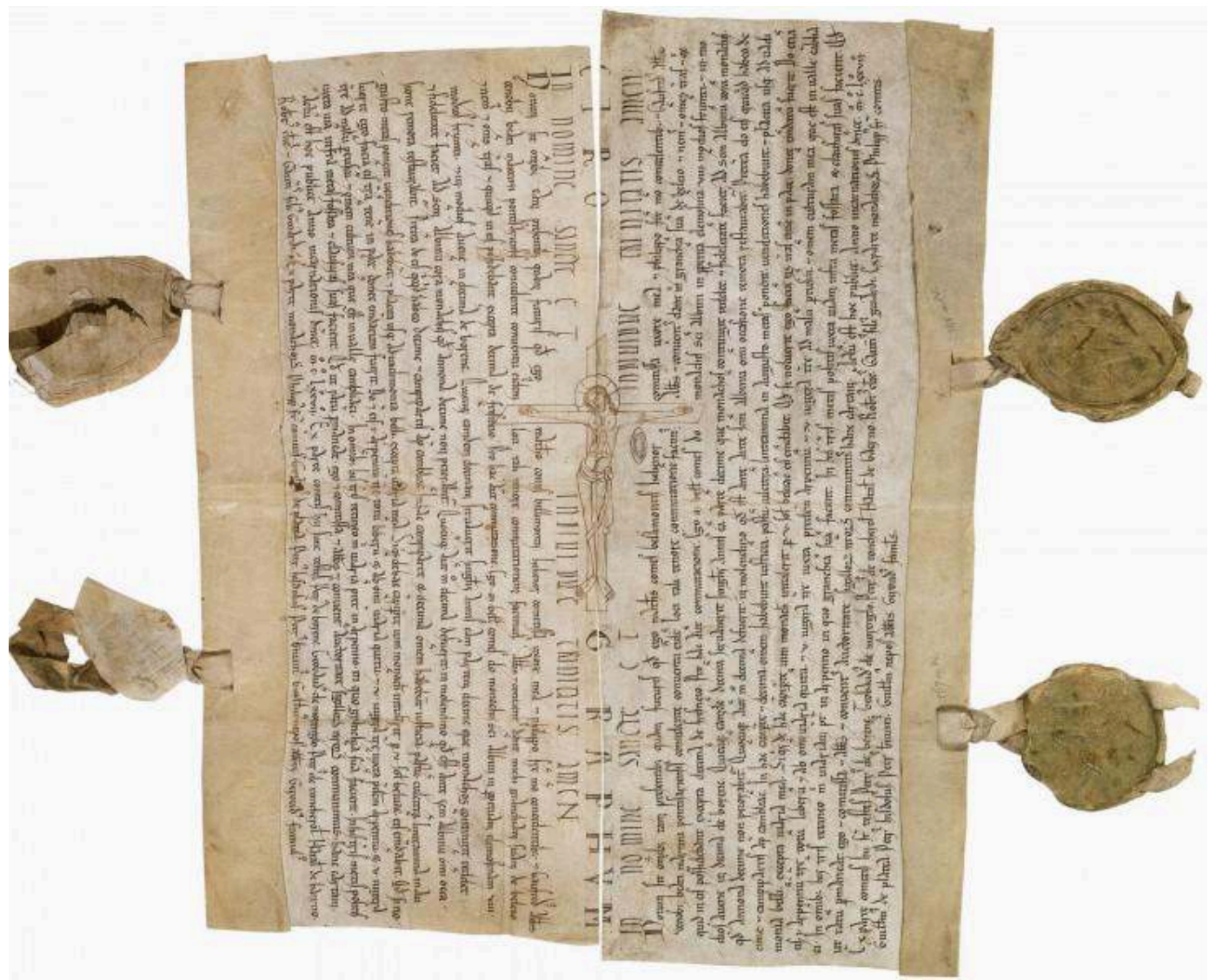

Chirographs are a medieval practice of authenticating documents, named from the practice of writing the word chirographum (with inconsistent orthography), which means "handwritten," between two identical copies of the same document and then cutting the two halves apart through the word, so that the parts can be recombined to show that the word lines up exactly. Actual practices vary, and need not include the word chirographum, but rely instead on images (see figure 6) or the shape of the cut: wavy cuts are called polls, jagged ("teeth-like") cuts are called indentures, and some chirographs might have three parts. A similar split-medium practice exists for account 
sticks, called tally sticks, which would be broken as a receipt, such that the splinters would uniquely match the account and prove that it had been paid. The medium is itself authenticating in a way that is simply not the case in ordinary documents. In figure 6, an example with both seals and chirograph attests to their shared use, as the authority of either half does not rest entirely upon the seals, but rather upon the status of each half lining up with its mate. In practice, chirographs, polls, and indentures circulated widely without seals, the split itself being sufficient authentication. Another authenticating medium is so-called "banknote paper," a special blend of different materials and fibers that is distinctive and can only be manufactured by specialized producers. An additional physical aspect of the medium, the watermark traditionally laid into paper, serves to identify the maker, and in the case of banknotes and other secure media serves to identify the paper as being made of the proper material (and presumably under the proper authority). Offices and individuals have long used basic authentication through physical media by writing or typing letters on institutional or individualized letterhead, controlling access to the stationery in order to protect its authority.

Going beyond the medium, there is a category of practices which combine physical characteristics and printing (spanning practices that are almost all the former to practices that are all the latter) in the service of authentication: security printing. This can include micrography (extremely small type that is hard to duplicate because cutting such fine dies is expensive) and unique typefaces with distinctive, purposeful irregularities that, owing to their very imperfections, are harder to duplicate than perfectly regular typefaces. It includes guilloche patterns, intricate and regular patterns that are hard to duplicate when cutting dies for printing. ${ }^{11}$ Other forms of security printing include the use of special inks, including inks designed to fluoresce in various wavelengths of light. Such inks are used in Canadian passports, where under UV light, Parliament Hill is surrounded by colorful fireworks, as it is on Canada Day. Following this chain of physical interventions in the service of authentication, one of the best ways to be assured that material is authentic is to receive it directly from a trusted source in a controlled manner. For a medieval document, a continuous chain of transmission through known and documented sources may suffice, as would continuous presence in a trusted and secure archival context. Ethiopian charters were often copied into the margins of "golden gospels" or other locally important manuscripts that would be well guarded by the local church and 
community. Presence in the margins of these sacred works ensured preservation and guarded against independently circulating forgeries. An example, from a copy of the Mäșhafä gizeyat(a lectionary with extracts from the Waddase Maryam, "Praise of Mary") of Zala 'Đnda 'Amanu'el Church in Tigray, has been discussed elsewhere, in terms of its clear documentary characteristics, despite not sharing much in material format with the typical single-page European charter (Wion 2018; Vogeler 2018). Similarly to storage in a locked safe, packaging or enclosure (where tamperresistant) assures the recipient that the item has been delivered in an unaltered condition. Security bags, security tape, tamper seals (on pharmaceuticals, for example), and lamination of documents all serve to establish the unaltered character of the material so enclosed, and therefore its unforged authenticity.

\section{5 A Generic Element: <authen>}

In order to clearly model all of these related practices, and in the interest of not favoring one period or type of document over another, a generic element seems both desirable and advisable. The proposed element, as implemented in the TEI_CEI ODD, ${ }^{12}$ is simple (attList items suppressed for brevity: they follow the top-level distinctions in the sample controlled vocabulary visualized in example 3):

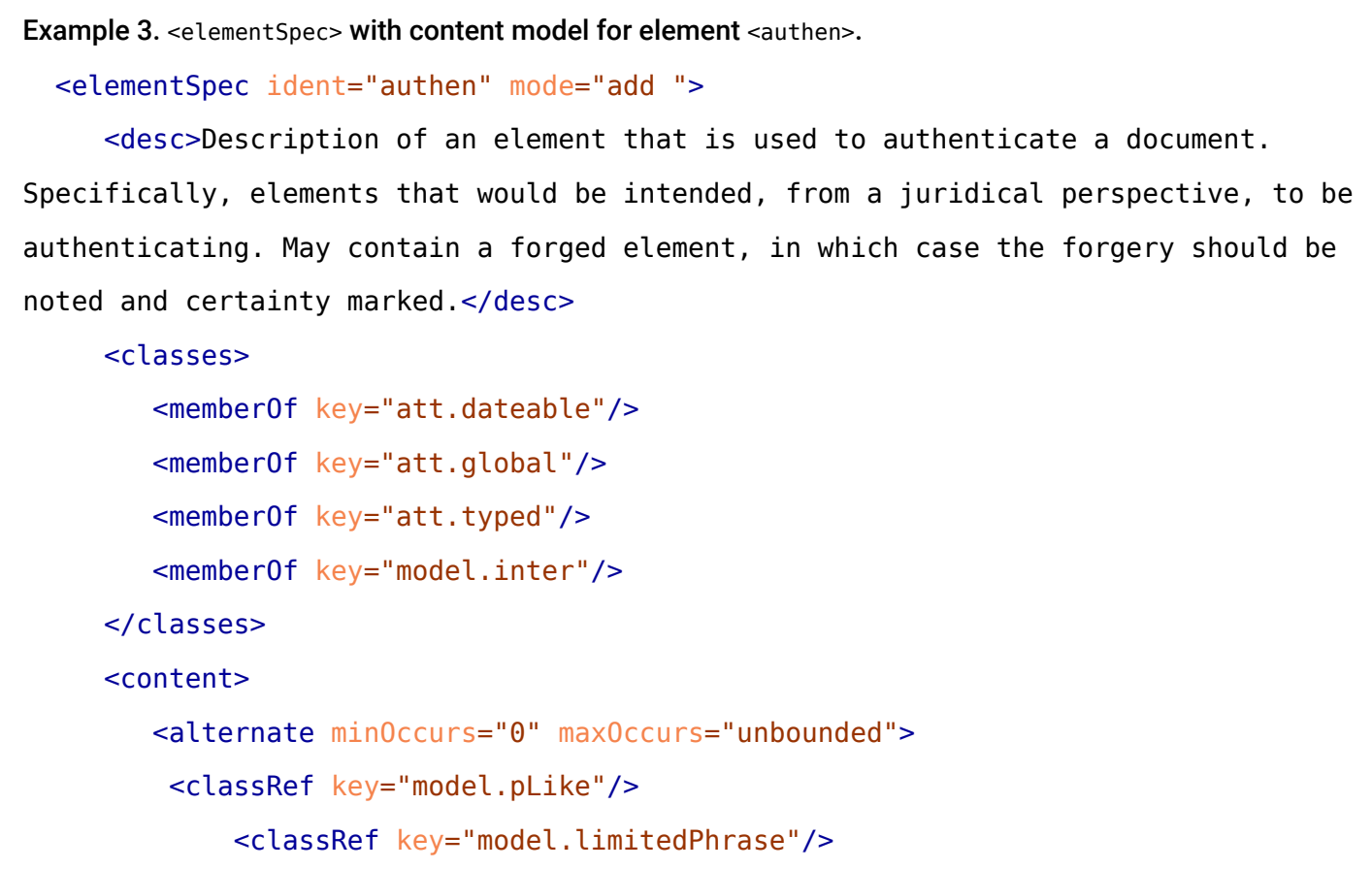




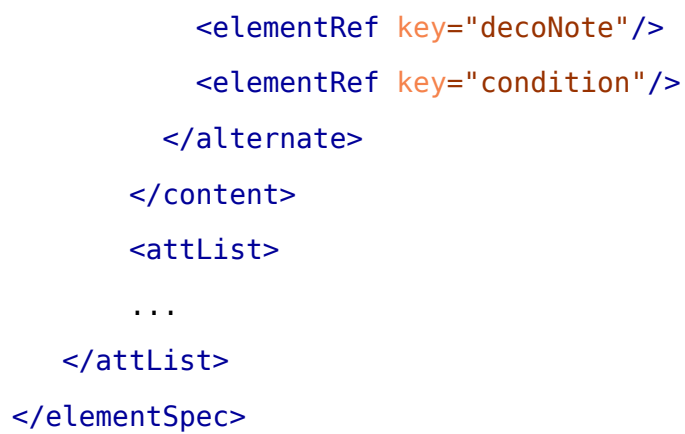

This feature genericizes the combination of physical/textual and paratextual elements in a seal to cover a whole range of authenticating features, serving not only the specific needs of charters encoding in the TEI, but also allowing for a fuller and more intercultural understanding of the nature of authenticating features that may be analogous to sealing in various contexts. By choosing to encode using <authen>, a richer, fuller, and more accurate view of the material can be encoded, as the intended context of these features (and with it the fullness of their import) is not discarded. It has a broader content model than <seal $>$, specifically model . LimitedPhrase, which is needed to cover all of the use cases of the CEI but also importantly captures model.pPart.msdesc, with a number of elements that are useful for describing seals themselves, as well as other types of authentication. Currently, this element is implemented in the TEI_CEI ODD for encoding charters, but the principles outlined here suggest that it should be available for broader use in the TEI. Alongside these changes, for the purposes of the TEI_CEI ODD, <sealDesc $>$ has been renamed to <authDesc>, to make the semantics consistent and understandable to the user. In order to control the wide range of different variants of this practice, here is a proposed vocabulary, provided in SKOS (Simple Knowledge Organization System) format (as part of the project's https://github.com/GVogeler/CEI2TEI/blob/master/Authentication/ authen.skos.ttlGitHub repository, ${ }^{13}$ at https://github.com/GVogeler/CEI2TEI/blob/master/ Authentication/authen.skos.ttl, which allows the identification of individual items of authentication from a large list. It is the product of a preliminary categorization of the various types of authentication that might appear in text-bearing objects encoded in the TEI P5. A visualization of this scheme, which was used in the preparation of the SKOS vocabulary, is reproduced in the appendix of this article. With the combination of this basic element and the wide range of potential subtype pointers (or equivalent modification of the ODD to enforce a project- 
specific attList), it should be possible to model a wide range of text-bearing documents, from ancient seals to modern banknotes and passports, as well as to enrich content-based markup of important paratextual authenticating elements.

24 It is my hope that this element, if picked up, will go some way toward allowing for more meaningful markup of a broad swath of transregional paratextual practices, helping TEI transcend some of its narrowly constructed limitations in the approach to such subjects from one specific regional/ disciplinary perspective. 
APPENDIXES

\section{Appendix 1. Addendum: Preliminary Work on Classification of Authenticating Features}

Figure 7. Visual representation of the organization behind the vocabulary of authenticating elements.

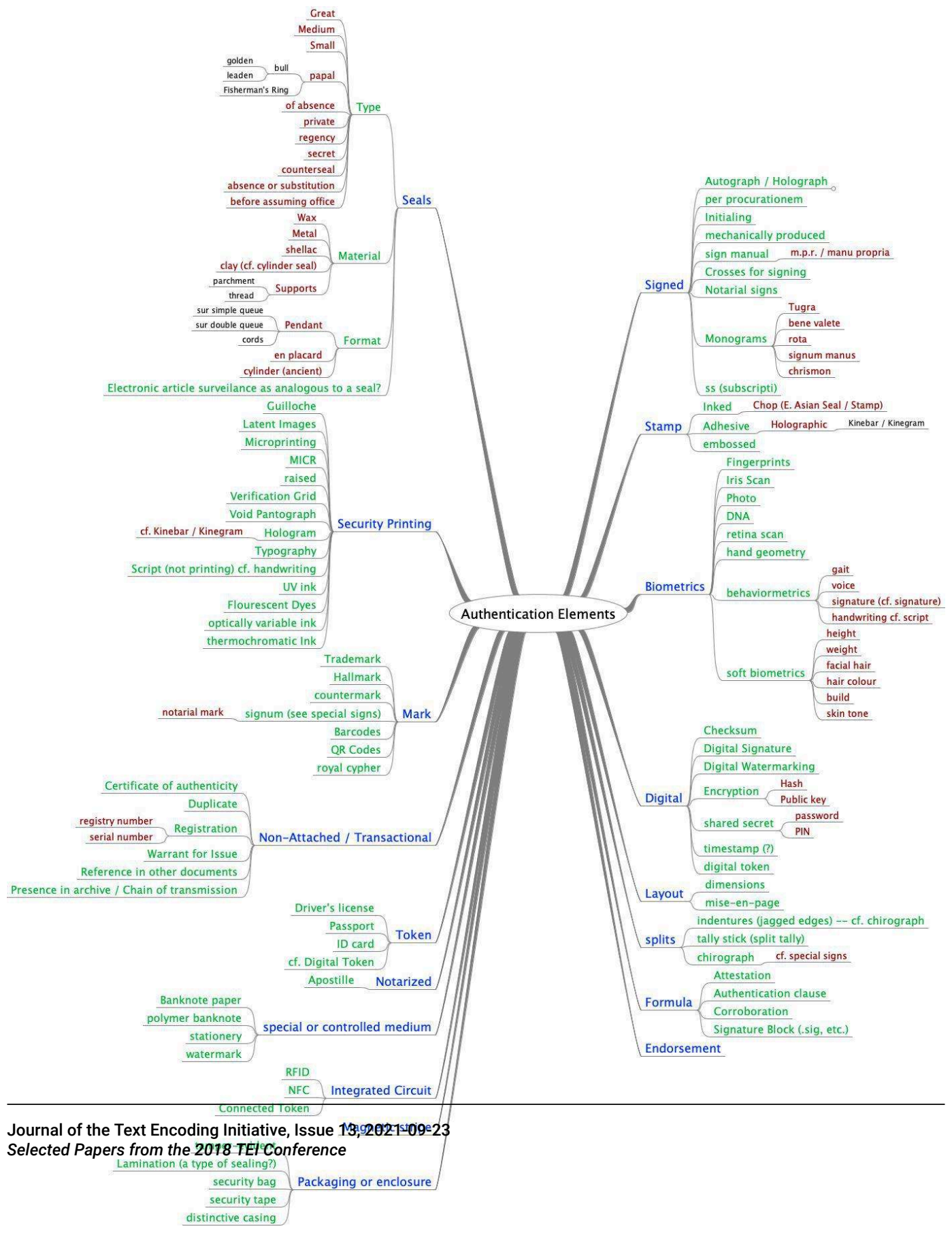




\section{BIBLIOGRAPHY}

“Biometrics." Android Open Source Project. Accessed December 4, 2018. https://source.android.com/ security/biometric.

Chango, Mawaki. 2012. "Becoming Artifacts: Medieval Seals, Passports and the Future of Digital Identity." PhD diss., Syracuse University. https://surface.syr.edu/it_etd/74.

Clemens, Raymond, and Timothy Graham. 2007. Introduction to Manuscript Studies.Ithaca, NY: Cornell University Press.

Collon, Dominique. 1987. First impressions: cylinder seals in the Ancient Near East.London: British Museum.

Cubelic, Simon, Axel Michaels, and Astrid Zotter, eds. 2018. Studies in Historical Documents from Nepal and India.Documenta Nepalica 1. Heidelberg: Heidelberg University Publishing. doi:10.17885/heiup.331.454.

Cummings, James. 2017. "Manuscript Description." Chap. 3 in Digital Editing of Medieval Texts: A Textbook,edited by Marjorie Burghart. N.p.: N.p. https://www.digitalmanuscripts.eu//wp-content/ uploads/2017/09/03-ms-desc-JC.pdf; https://www.digitalmanuscripts.eu/?page_id=648.

Mabillon, Jean. 1681. De re diplomatica libri VI.Paris.

“Monumenta Germaniae Historica: Constitutiones et acta publica imperatorum et regum 1357-1378." 2019. Berlin-Brandenburgische Akademie der Wissenschaften. Accessed July 22. http://telota.bbaw.de/ constitutiones/index.html.

New, Elizabeth A. 2010. Seals and Sealing Practices. London: British Records Association.

Ramble, Charles. 2018. “Producing ‘South Asian’ Tibetan Documents in Highland Nepal, 19th to 20th Centuries: The Emergence and Disappearance of a Local Diplomatic Tradition." In Cubelic, Michaels, and Zotter $2018,111-33$.

Rück, Peter, ed. 1996. Graphische Symbole in mittelalterlichen Urkunden: Beiträge zur diplomatischen Semiotik. Historische Hilfswissenschaften 3. Sigmaringen: Jan Thorbecke.

Sopracasa, Alessio, and Martina Filosa. 2020. "Encoding Byzantine Seals: SigiDoc," in Atti del IX Convegno Annuale AIUCD. La svolta inevitabile: sfide e prospettive perl'Informatica Umanistica, 240-45. Milano: Università Cattolica del Sacro Cuore. http://aiucd2020.unicatt.it/aiucd-Sopracasa_Filosa.pdf.

TEI Consortium. 2019. TEI P5: Guidelines for Electronic Text Encoding and Interchange. Version 3.6.0. Last updated July 19. N.p.: TEI Consortium. https://tei-c.org/Vault/P5/3.6.0/doc/tei-p5-doc/en/html/.

“Use Touch ID on your Mac."MacOS User Guide. Apple Inc. Accessed December 4, 2018. https:// support.apple.com/en-us/HT207054.

Valla, Lorenzo. 1440. De falso credita et ementita Constantini Donatione declamatio. 
Vogeler, Georg. 2018. “Digital Diplomatics: The Evolution of a European Tradition or a Generic Concept?” In Cubelic, Michaels, and Zotter 2018, 85-109.

Vogeler, Georg, et al. 2018. CEI - Charters Encoding Initiative. Accessed December 4. https://www.cei.lmu.de. Winslow, Sean M., and Georg Vogeler. 2019. CEI2TEI.Github. Last revised June 21. https://github.com/ GVogeler/CEI2TEI.

Wion, Anaïs. 2018. "The TEI-XML Architecture of Ethiopian Manuscript Archives: Respecting the Integrity of Primary Sources and Asserting Editorial Choices." Comparative Oriental Manuscript Studies Bulletin4 (1): 3338. https://www.aai.uni-hamburg.de/en/comst/pdf/bulletin4-1/33-38.pdf.

\section{NOTES}

1 CEI website, accessed June 25, 2021, http://www.cei.lmu.de/.

2 I am also grateful for the advice and feedback of the anonymous reviewers of this paper.

3 "Nomisma Ontology," accessed July 1, 2021, http://nomisma.org/ontology.

4 I leave aside here the question of forged authentication, which is outside the limited scope of this paper, and, in any event, could be dealt with appropriately using existing markup.

5 The concept of authentication as witness to something both being true and being legal is discussed by Chango (2012).

6 A useful (though UK-centric) introduction to sealing practices is by New (2010).

7 From about 3400 BCE. Collon 1987.

$8<\mathrm{p}>$, in turn, has a larger number of descendants, but the point is that these essentially equivalent features are modeled in such a way that they have no direct overlap in child elements.

9 Monumenta Germaniae Historica: Constitutiones et acta publica imperatorum et regum 1357-1378, BerlinBrandenburgische Akademie der Wissenschaften, accessed July 22, 2019, http://telota.bbaw.de/ constitutiones/index.html.

10 Going beyond medieval examples, a technology which also uses fingerprints, biometric identity management, once the purview of science fiction, has become increasingly common in personal computing devices: iOS and Android devices, among others, support fingerprint or facial recognition technology, supplementing traditional passwords ("Use Touch ID on your Mac" n.d.; 
"Biometrics" n.d.). Future technology may include retina or iris scans (already in use at airport gates), fingerprints (associated with biometric passports), and other biometric data associated with a record in order to establish its identity.

11 After all, printed type and printed banknotes are produced through functionally the same process as stamping a document, and the use of dies differs little from pressing a matrix into wax to seal it.

12 See the CEI2TEI GitHub repository, accessed June 25, 2021, https:/github.com/GVogeler/ CEI2TEI/blob/master/tei_cei.odd.

13 Accessed July 13, 2021, https://github.com/GVogeler/CEI2TEI/blob/master/Authentication/ authen.skos.ttl.

\section{AUTHOR}

\section{SEAN M. WINSLOW}

Postdoc on the FWF (Austrian Science Fund) project Retain Domain Specific Functionalities in a Generic Repository with Humanities Data (ORD84), Karl-Franzens-Universität Graz 